\title{
Boundary Stabilization of the Wave Equation with Time-Varying and Nonlinear Feedback
}

\author{
Jian-Sheng Tian,, Wei Wang, ${ }^{1,2}$ Fei Xue, ${ }^{1,2}$ and Pei-Yong Cong ${ }^{1,2}$ \\ ${ }^{1}$ College of Computer Science, Beijing University of Technology, Beijing 100124, China \\ ${ }^{2}$ State Engineering Laboratory of Information System Classified Protection Key Technologies, Beijing University of Technology, \\ Beijing 100124, China
}

Correspondence should be addressed to Jian-Sheng Tian; jian-sheng.tian@outlook.com

Received 24 April 2014; Accepted 7 June 2014; Published 29 June 2014

Academic Editor: Yoshinori Hayafuji

Copyright (C) 2014 Jian-Sheng Tian et al. This is an open access article distributed under the Creative Commons Attribution License, which permits unrestricted use, distribution, and reproduction in any medium, provided the original work is properly cited.

We study the stabilization of the wave equation with variable coefficients in a bounded domain and a time-varying and nonlinear term. By the Riemannian geometry methods and a suitable assumption of nonlinearity and the time-varying term, we obtain the uniform decay of the energy of the system.

\section{Introduction}

There are many results concerning the boundary stabilization of classical wave equations. See [1-6] for linear cases and [712] for nonlinear ones. The stability of the wave equation with variable coefficients has attracted much attention. See [13$23]$, and many others. In [20], by the methods in [11, 24], the authors study the stability of the wave equation with nonlinear term and time-varying term. However, under the condition the nonlinear term has upper bound and the timevarying term has lower bound, the stability of the wave equation was not studied in [20]. In this paper, our purpose is to study the stability of the wave equation under the condition the nonlinear term has upper bound and the time-varying term has lower bound.

Let $\Omega$ be a bounded domain in $\mathbb{R}^{n}$ with smooth boundary $\Gamma$. It is assumed that $\Gamma$ consists of two parts $\Gamma_{1}$ and $\Gamma_{2}(\Gamma=$ $\Gamma_{1} \cup \Gamma_{2}$ ) with $\Gamma_{2} \neq \emptyset, \bar{\Gamma}_{1} \cap \bar{\Gamma}_{2}=\emptyset$. Define

$$
\mathscr{A} u=-\operatorname{div} A(x) \nabla u \quad \text { for } u \in H^{1}(\Omega),
$$

where div is the divergence operator of the standard metric of $\mathbb{R}^{n} ; A(x)=\left(a_{i j}(x)\right)$ is symmetric, positively definite matrices for each $x \in \mathbb{R}^{n}$ and $a_{i j}(x)$ are smooth functions on $\mathbb{R}^{n}$.
We consider the stabilization of the wave equations with variable coefficients and time-varying delay in the dissipative boundary feedback:

$$
\begin{gathered}
u_{t t}+\mathscr{A} u=0 \quad(x, t) \in \Omega \times(0,+\infty), \\
\left.u(x, t)\right|_{\Gamma_{2}}=0 \quad t \in(0,+\infty), \\
\frac{\partial u(x, t)}{\partial v_{\mathscr{A}}}+\phi(t) g_{1} u_{t}(x, t)=0 \quad(x, t) \in \Gamma_{1} \times \in(0,+\infty), \\
u(x, 0)=u_{0}(x), \quad u_{t}(x, 0)=u_{1}(x) \quad x \in \Omega,
\end{gathered}
$$

$g_{1} \in C(\mathbb{R})$ and there exists a positive constant $c_{1}$ such that

$$
\begin{gathered}
g_{1}(0)=0, \quad s g_{1}(s) \leq|s|^{2} \quad \text { for } s \in \mathbb{R} \\
\left|g_{1}(s)\right| \geq c_{1}|s| \quad \text { for }|s|>1,
\end{gathered}
$$

and $\phi(t) \in C([0,+\infty))$ satisfies

$$
\begin{gathered}
\phi(t) \geq \phi_{0} \quad \forall t \geq 0, \\
\lim _{t \rightarrow+\infty} \frac{F(t)}{t}=0,
\end{gathered}
$$

where $\phi_{0}$ is a positive constant and $F(t)=\max _{0 \leq \rho \leq t} \phi(\rho)$. 
$\partial u / \partial v_{\mathscr{A}}$ is the conormal derivative

$$
\frac{\partial u}{\partial \nu_{\mathscr{A}}}=\langle A(x) u, v\rangle
$$

where $\langle\cdot, \cdot\rangle$ denotes the standard metric of the Euclidean space $\mathbb{R}^{n}$ and $\nu(x)$ is the outside unit normal vector for each $x \in \Gamma$. Moreover, the initial data $\left(u_{0}, u_{1}\right)$ belongs to a suitable space.

Define the energy of the system (2) by

$$
E(t)=\frac{1}{2} \int_{\Omega}\left(u_{t}^{2}+\sum_{i, j=1}^{n} a_{i j} u_{x_{i}} u_{x_{j}}\right) d x .
$$

We define

$$
g=A^{-1}(x) \quad \text { for } x \in \mathbb{R}^{n}
$$

as a Riemannian metric on $\mathbb{R}^{n}$ and consider the couple $\left(\mathbb{R}^{n}, g\right)$ as a Riemannian manifold with an inner product:

$$
\langle X, Y\rangle_{g}=\left\langle A^{-1}(x) X, Y\right\rangle, \quad|X|_{g}^{2}=\langle X, X\rangle_{g} \quad X, Y \in \mathbb{R}_{x}^{n} .
$$

Let $D_{g}$ denote the Levi-Civita connection of the metric $g$. For the variable coefficients, the main assumptions are as follows.

Assumption $A$. There exists a vector field $H$ on $\bar{\Omega}$ and a constant $\rho_{0}>0$ such that

$$
D_{g} H(X, X) \geq \rho_{0}|X|_{g}^{2} \quad \text { for } X \in \mathbb{R}_{x}^{n}, x \in \bar{\Omega} .
$$

Moreover we assume that

$$
\begin{gathered}
\sup _{x \in \bar{\Omega}} \operatorname{div} H<\inf _{x \in \bar{\Omega}} \operatorname{div} H+2 \rho_{0}, \\
H \cdot v \leq 0 \quad x \in \Gamma_{2}, \\
H \cdot v \geq \delta \quad x \in \Gamma_{1},
\end{gathered}
$$

where $\delta$ is a positive constant.

Assumption (10) was introduced by [13] as a checkable assumption for the exact controllability of the wave equation with variable coefficients. For examples on the condition, see $[13,14]$.

Based on Assumption (10), Assumption A was given by [19] to study the stabilization of the wave equation with variable coefficients and boundary condition of memory type.

Define

$$
H_{\Gamma_{2}}^{1}(\Omega)=\left\{u \in H^{1}(\Omega)|u|_{\Gamma_{2}}=0\right\} .
$$

To obtain the stabilization of the system (2), we assume the system (2) is well-posed such that

$$
u \in C^{1}\left([0,+\infty), L^{2}(\Omega)\right) \cap C\left([0,+\infty), H_{\Gamma_{2}}^{1}(\Omega)\right) .
$$

The main result of this paper is stated as follows.

Theorem 1. Let Assumption A holds true. Then there exist positive constants $C, C_{2}$, such that

$$
E(t) \leq C_{1} h\left(\frac{C_{2} E(0)}{t}\right)+\frac{C_{1} F(t)}{t} E(0), \quad t>0 .
$$

\section{Basic Inequality of the System}

In this section we work on $\Omega$ with two metrics at the same time, the standard dot metric $\langle\cdot, \cdot\rangle$ and the Riemannian metric $g=\langle\cdot, \cdot\rangle_{g}$ given by $(8)$.

If $f \in C^{1}\left(\mathbb{R}^{n}\right)$, we define the gradient $\nabla_{g} f$ of $f$ in the Riemannian metric $g$, via the Riesz representation theorem, by

$$
X(f)=\left\langle\nabla_{g} f, X\right\rangle_{g}
$$

where $X$ is any vector field on $\left(\mathbb{R}^{n}, g\right)$. The following lemma provides further relations between the two metrics; see [13] in Lemma 2.1.

Lemma 2. Let $x=\left(x_{1}, \ldots, x_{n}\right)$ be the natural coordinate system in $\mathbb{R}^{n}$. Let $f, h$ be functions and let $\mathscr{H}, X$ be vector fields. Then

(a)

$$
\langle H(x), A(x) X(x)\rangle_{g}=\langle H(x), X(x)\rangle, \quad x \in \mathbb{R}^{n} ;
$$

(b)

$$
\nabla_{g} f=\sum_{i=1}^{n}\left(\sum_{j=1}^{n} a_{i j}(x) f_{x_{j}}\right) \frac{\partial}{\partial x_{i}}=A(x) \nabla f, \quad x \in \mathbb{R}^{n},
$$

where $\nabla f$ is the gradient of $f$ in the standard metric;

(c)

$$
\nabla_{g} f(h)=\left\langle\nabla_{g} f, \nabla_{g} h\right\rangle_{g}=\langle\nabla f, A(x) \nabla h\rangle, \quad x \in \mathbb{R}^{n},
$$

where the matrix $A(x)$ is given in formula (1). Define

To prove Theorem 1, we still need several lemmas further.

$$
E_{0}(t)=\frac{1}{2} \int_{\Omega}\left(u_{t}^{2}+\left|\nabla_{g} u\right|_{g}^{2}\right) d x
$$

Then, we have

$$
E(t)=E_{0}(t)+\xi \int_{t-\tau(t)}^{t} \int_{\Gamma_{1}} u_{t}^{2}(x, \rho) d \Gamma d \rho .
$$

Lemma 3. Let $(u)$ be the solution of system (2). Then there exists a constant $C_{1}$ such that

$$
E(0)-E(T)=C_{1} \int_{0}^{T} \int_{\Gamma_{1}} \phi(t) u_{t}(x, t) g_{1}\left(u_{t}(x, t)\right) d \Gamma d t
$$

where $T \geq 0$. The assertion (22) implies that $E(t)$ is decreasing.

Proof. Differentiating (7), we obtain

$$
\begin{aligned}
E^{\prime}(t) & =\int_{\Omega}\left(u_{t} u_{t t}+\nabla_{g} u \cdot \nabla u_{t}\right) d x \\
& =\int_{\Gamma_{1}} \phi(t) u_{t}(x, t) g_{1}\left(u_{t}(x, t)\right) d \Gamma .
\end{aligned}
$$

Then the inequality (22) holds true. 


\section{Proofs of Theorem 1}

From Proposition 2.1 in [13], we have the following identities.

Lemma 4. Suppose that $u(x, t)$ solves equation $u_{t t}+\mathscr{A} u=0$, $(x, t) \in \Omega \times(0,+\infty)$ and that $\mathscr{H}$ is a vector field defined on $\bar{\Omega}$. Then, for $T \geq 0$,

$$
\begin{aligned}
& \int_{0}^{T} \int_{\Gamma} \frac{\partial u}{\partial v_{\mathscr{A}}} \mathscr{H}(u) d \Gamma d t \\
& \quad+\frac{1}{2} \int_{0}^{T} \int_{\Gamma}\left(u_{t}^{2}-\left|\nabla_{g} u\right|_{g}^{2}\right) \mathscr{H} \cdot v d \Gamma d t \\
& =\left.\left(u_{t}, \mathscr{H}(u)\right)\right|_{0} ^{T}+\int_{0}^{T} \int_{\Omega} D_{g} \mathscr{H}\left(\nabla_{g} u, \nabla_{g} u\right) d x d t \\
& \quad+\frac{1}{2} \int_{0}^{T} \int_{\Omega}\left(u_{t}^{2}-\left|\nabla_{g} u\right|_{g}^{2}\right) \operatorname{div} \mathscr{H} d x d t .
\end{aligned}
$$

Moreover, assume that $P \in C^{1}(\bar{\Omega})$. Then

$$
\begin{aligned}
\int_{0}^{T} \int_{\Omega}\left(u_{t}^{2}-\left|\nabla_{g} u\right|_{g}^{2}\right) P d x d t \\
=\left.\left(u_{t}, u P\right)\right|_{0} ^{T}+\frac{1}{2} \int_{0}^{T} \int_{\Omega} \nabla_{g} P\left(u^{2}\right) d x d t \\
\quad-\int_{0}^{T} \int_{\Gamma} P u \frac{\partial u}{\partial v_{\mathscr{A}}} d \Gamma d t .
\end{aligned}
$$

Lemma 5. Suppose that all assumptions in Theorem 1 hold true. Let $u$ be the solution of the system (2). Then there exist positive constants $C, T_{0}$ for which

$$
E(T) \leq \frac{C}{T} \int_{0}^{T} \int_{\Gamma_{1}}\left(u_{t}^{2}+\left(\frac{\partial u}{\partial \nu_{\mathscr{A}}}\right)^{2}\right) d \Gamma d t,
$$

where $T \geq T_{0}$.

Proof. We let $\theta$ be a positive constant satisfying

$$
\frac{1}{2} \sup _{x \in \bar{\Omega}} \operatorname{div} H<\theta<\frac{1}{2} \inf _{x \in \bar{\Omega}} \operatorname{div} H+\rho_{0} .
$$

Set

$$
\mathscr{H}=H, \quad P=\theta-\rho_{0} .
$$

Substituting the identity (25) into the identity (24), we obtain

$$
\begin{aligned}
\Pi_{\Gamma}= & \left.\left(u_{t}, H(u)+P u\right)\right|_{0} ^{T} \\
& +\int_{0}^{T} \int_{\Omega}\left(D_{g} H\left(\nabla_{g} u, \nabla_{g} u\right)-\rho_{0}\left|\nabla_{g} u\right|_{g}^{2}\right) d x d t \\
& +\int_{0}^{T} \int_{\Omega}\left(\left(\frac{1}{2} \operatorname{div} H+\rho_{0}-\theta\right) u_{t}^{2}\right. \\
& \left.+\left(\theta-\frac{1}{2} \operatorname{div} H\right)\left|\nabla_{g} u\right|_{g}^{2}\right) d x d t,
\end{aligned}
$$

where

$$
\begin{aligned}
\Pi_{\Gamma}= & \int_{0}^{T} \int_{\Gamma} \frac{\partial u}{\partial v_{\mathscr{A}}}(H(u)+u P) d \Gamma d t \\
& +\frac{1}{2} \int_{0}^{T} \int_{\Gamma}\left(u_{t}^{2}-\left|\nabla_{g} u\right|_{g}^{2}\right) H \cdot v d \Gamma d t .
\end{aligned}
$$

Decompose $\Pi_{\Gamma}$ as

$$
\Pi_{\Gamma}=\Pi_{\Gamma_{1}}+\Pi_{\Gamma_{2}}
$$

Since $\left.u\right|_{\Gamma_{2}}=0$, we obtain $\left.\nabla_{\Gamma} u\right|_{\Gamma_{2}}=0$; that is,

$$
\nabla_{g} u=\frac{\partial u}{\partial v_{\mathscr{A}}} \frac{v_{\mathscr{A}}}{\left|v_{\mathscr{A}}\right|_{\mathscr{g}}^{2}} \quad \text { for } x \in \Gamma_{2} \text {. }
$$

Similarly, we have

$$
H(u)=\left\langle H, \nabla_{g} u\right\rangle_{g}=\frac{\partial u}{\partial \nu_{\mathscr{A}}} \frac{H \cdot v}{\left|\nu_{\mathscr{A}}\right|_{g}^{2}} \quad \text { for } x \in \Gamma_{2} .
$$

Using the formulas (32) and (33) in the formula (30) on the portion $\Gamma_{2}$, with (12), we obtain

$$
\Pi_{\Gamma_{2}}=\frac{1}{2} \int_{0}^{T} \int_{\Gamma_{2}}\left(\frac{\partial u}{\partial \nu_{\mathscr{A}}}\right)^{2} \frac{H \cdot v}{\left|\nu_{\mathscr{A}}\right|_{g}^{2}} d \Gamma d t \leq 0 .
$$

From (12), we have

$$
\begin{aligned}
\Pi_{\Gamma_{1}}= & \int_{0}^{T} \int_{\Gamma_{1}} \frac{\partial u}{\partial v_{\mathscr{A}}}(H(u)+u P) d \Gamma d t \\
& +\frac{1}{2} \int_{0}^{T} \int_{\Gamma_{1}}\left(u_{t}^{2}-\left|\nabla_{g} u\right|_{g}^{2}\right) H \cdot v d \Gamma d t \\
\leq & C_{\varepsilon} \int_{0}^{T} \int_{\Gamma_{1}}\left(\frac{\partial u}{\partial \nu_{\mathscr{A}}}\right)^{2} d \Gamma d t \\
& +\varepsilon \int_{0}^{T} \int_{\Gamma_{1}}\left(u^{2}+\left|\nabla_{g} u\right|_{g}^{2}\right) d \Gamma d t \\
& +\int_{0}^{T} \int_{\Gamma_{1}}\left(C u_{t}^{2}-\delta\left|\nabla_{g} u\right|_{g}^{2}\right) d \Gamma d t \\
\leq & C \int_{0}^{T} \int_{\Gamma_{1}}\left(\frac{\partial u}{\partial \nu_{\mathscr{A}}}\right)^{2} d \Gamma d t+\varepsilon E(t) \\
& +C \int_{0}^{T} \int_{\Gamma_{1}} u_{t}^{2} d \Gamma d t .
\end{aligned}
$$

Substituting the formulas (34) and (35) into the formula (29), with (27), we obtain

$$
\begin{aligned}
& \int_{0}^{T} E(t) d t \\
& \quad \leq C(E(0)+E(T))+C \int_{0}^{T} \int_{\Gamma_{1}}\left(u_{t}^{2}+\left(\frac{\partial u}{\partial \nu_{\mathscr{A}}}\right)^{2}\right) d \Gamma d t .
\end{aligned}
$$


It follows from (22) that

$$
\int_{0}^{T} E(t) d t \geq T E(T)
$$

Substituting the formulas (22) and (37) into the formula (36), the inequality (26) holds.

Proof of Theorem 1. Since $E(t)$ is decreasing, with (4) and (26), for sufficiently large $T$, we have

$$
\begin{aligned}
& E(T) \\
& \leq \frac{C}{T} \int_{0}^{T} \int_{\Gamma_{1}}\left(\phi^{2}(t) g^{2}\left(u_{t}\right)+u_{t}^{2}\right) d \Gamma d t \\
& \leq \frac{C}{T}\left\{\int_{0}^{T} \int_{\Gamma_{1}}\left(u_{t}^{2}+g^{2}\left(u_{t}\right)\right) d \Gamma d t\right. \\
& \left.\leq \quad+F(T) \int_{0}^{T} \int_{\Gamma_{1}} u_{t} \phi(t) g\left(u_{t}\right) d \Gamma d t\right\} \\
& \leq \frac{C}{T} \int_{0}^{T} \int_{\Gamma_{1}}^{T} h\left(u_{t} g\left(u_{t}\right)\right) d \Gamma d t+\frac{C F(T)}{T} E(0) \\
& \leq C \text { meas }\left(\Gamma_{1}\right) h\left(\frac{\left.\int_{0}, u_{t} \mid \leq 1\right\}}{\Gamma_{1}} u_{t} g\left(u_{t} g\left(u_{t}\right)\right) d \Gamma d t+C F(T) E(0)\right\} \\
& \leq C, h\left(\frac{C_{2} E(0)}{T}\right)+\frac{C_{1} F(T)}{T} E(0) .
\end{aligned}
$$

Note that $E(t)$ is decreasing; the estimate (15) holds.

\section{Application of the System (2)}

Nonlinear feedback describes a property of a physical system; that is, the response by the physical system to an applied force is nonlinear in its effect. One of the applications of the system (2) is in sound waves, where the system (2) describes the reflection of sound in heterogeneous materials at surfaces of some materials with nonlinearity of interest in engineering practice. Theorem 1 indicates that the energy of the sound waves with the reflection of sound at surfaces in heterogeneous materials at surfaces of some materials with nonlinearity is uniform decay under a suitable assumption of the nonlinearity.

\section{Conflict of Interests}

The authors declare that there is no conflict of interests regarding the publication of this paper.

\section{References}

[1] J. E. Lagnese, "Note on boundary stabilization of wave equations," SIAM Journal on Control and Optimization, vol. 26, no. 5, pp. 1250-1256, 1988.

[2] D. L. Russell, "Controllability and stabilizability theory for linear partial differential equations: recent progress and open questions," SIAM Review, vol. 20, no. 4, pp. 639-739, 1978.

[3] R. Triggiani, "Wave equation on a bounded domain with boundary dissipation: an operator approach," Journal of Mathematical Analysis and Applications, vol. 137, no. 2, pp. 438-461, 1989.

[4] Y. You, "Energy decay and exact controllability for the Petrovsky equation in a bounded domain," Advances in Applied Mathematics, vol. 11, no. 3, pp. 372-388, 1990.

[5] M. Aassila, M. M. Cavalcanti, and V. N. D. Cavalcanti, "Existence and uniform decay of the wave equation with nonlinear boundary damping and boundary memory source term," Calculus of Variations and Partial Differential Equations, vol. 15, no. 2, pp. 155-180, 2002.

[6] M. M. Cavalcanti, V. N. Domingos Cavalcanti, and P. Martinez, "Existence and decay rate estimates for the wave equation with nonlinear boundary damping and source term," Journal of Differential Equations, vol. 203, no. 1, pp. 119-158, 2004.

[7] F. Conrad and B. Rao, "Decay of solutions of the wave equation in a star-shaped domain with nonlinear boundary feedback," Asymptotic Analysis, vol. 7, no. 3, pp. 159-177, 1993.

[8] V. Komornik, "On the nonlinear boundary stabilization of the wave equation," Chinese Annals of Mathematics B, vol. 14, no. 2, pp. 153-164, 1993.

[9] V. Komornik, Exact Controllability and Stabilization: The Multiplier Method, John Wiley \& Sons, Chichester, UK, 1994.

[10] V. Komornik and E. Zuazua, "A direct method for the boundary stabilization of the wave equation," Journal de Mathématiques Pures et Appliquées, vol. 69, no. 1, pp. 33-54, 1990.

[11] I. Lasiecka and D. Tataru, "Uniform boundary stabilization of semilinear wave equations with nonlinear boundary damping," Differential and Integral Equations, vol. 6, no. 3, pp. 507-533, 1993.

[12] E. Zuazua, "Uniform stabilization of the wave equation by nonlinear boundary feedback," SIAM Journal on Control and Optimization, vol. 28, no. 2, pp. 466-477, 1990.

[13] P. F. Yao, "On the observability inequalities for exact controllability of wave equations with variable coefficients," SIAM Journal on Control and Optimization, vol. 37, no. 5, pp. 15681599, 1999.

[14] P.-F. Yao, Modeling and Control in Vibrational and Structural Dynamics: A Differential Geometric Approach, Chapman \& Hall/ CRC Applied Mathematics and Nonlinear Science Series, CRC Press, Boca Raton, Fla, USA, 2011.

[15] I. Lasiecka, R. Triggiani, and P. F. Yao, "Inverse/observability estimates for second-order hyperbolic equations with variable coefficients," Journal of Mathematical Analysis and Applications, vol. 235, no. 1, pp. 13-57, 1999.

[16] Z. H. Ning and Q. X. Yan, "Stabilization of the wave equation with variable coefficients and a delay in dissipative boundary feedback," Journal of Mathematical Analysis and Applications, vol. 367, no. 1, pp. 167-173, 2010.

[17] Z. H. Ning, C. X. Shen, and X. P. Zhao, "Stabilization of the wave equation with variable coefficients and a delay in dissipative internal feedback," Journal of Mathematical Analysis and Applications, vol. 405, no. 1, pp. 148-155, 2013. 
[18] Z. H. Ning, C. X. Shen, X. Zhao, H. Li, C. Lin, and Y. M. Zhang, "Nonlinear boundary stabilization of the wave equations with variable coefficients and time dependent delay," Applied Mathematics and Computation, vol. 232, pp. 511-520, 2014.

[19] S. Nicaise and C. Pignotti, "Stabilization of the wave equation with variable coefficients and boundary condition of memory type," Asymptotic Analysis, vol. 50, no. 1-2, pp. 31-67, 2006.

[20] B. Gong and X. Zhao, "Boundary stabilization of a semilinear wave equation with variable coefficients under the time-varying and nonlinear feedback," Abstract and Applied Analysis, vol. 2014, Article ID 728760, 6 pages, 2014.

[21] H. Li, C. S. Lin, S. P. Wang, and Y. M. Zhang, "Stabilization of the wave equation with boundary time-varying delay," Advances in Mathematical Physics, vol. 2014, Article ID 735341, 6 pages, 2014.

[22] B.-Z. Guo and Z.-C. Shao, "On exponential stability of a semilinear wave equation with variable coefficients under the nonlinear boundary feedback," Nonlinear Analysis: Theory, Methods \& Applications, vol. 71, no. 12, pp. 5961-5978, 2009.

[23] Z. H. Ning, C. X. Shen, and X. P. Zhao, "stabilization of the wave equation with variable coefficients and a internal memory type," Nonlinear Analysis: Real World Applications. In press.

[24] M. M. Cavalcanti, V. N. D. Cavalcanti, and I. Lasiecka, "Wellposedness and optimal decay rates for the wave equation with nonlinear boundary damping-source interaction," Journal of Differential Equations, vol. 236, no. 2, pp. 407-459, 2007. 


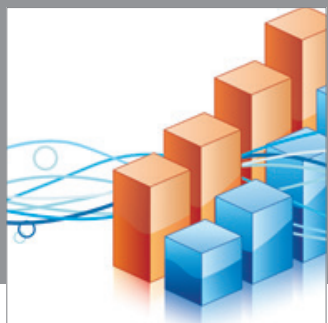

Advances in

Operations Research

mansans

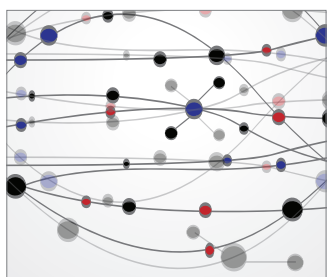

The Scientific World Journal
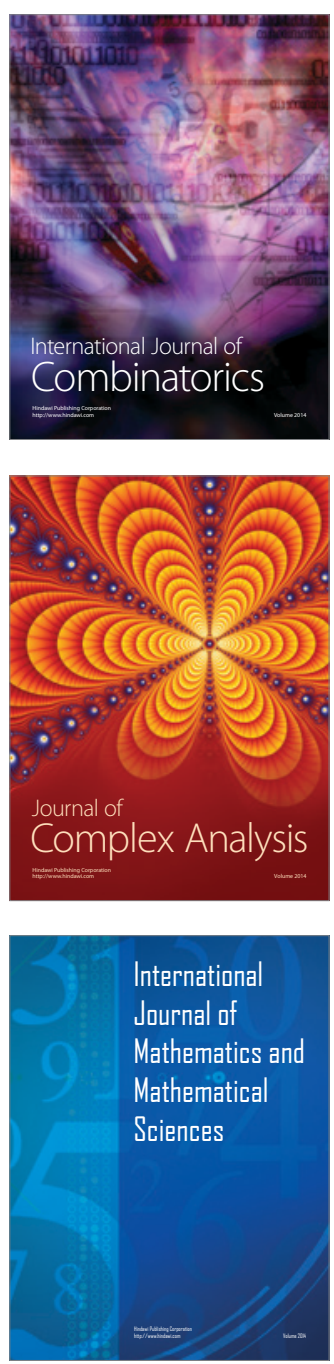
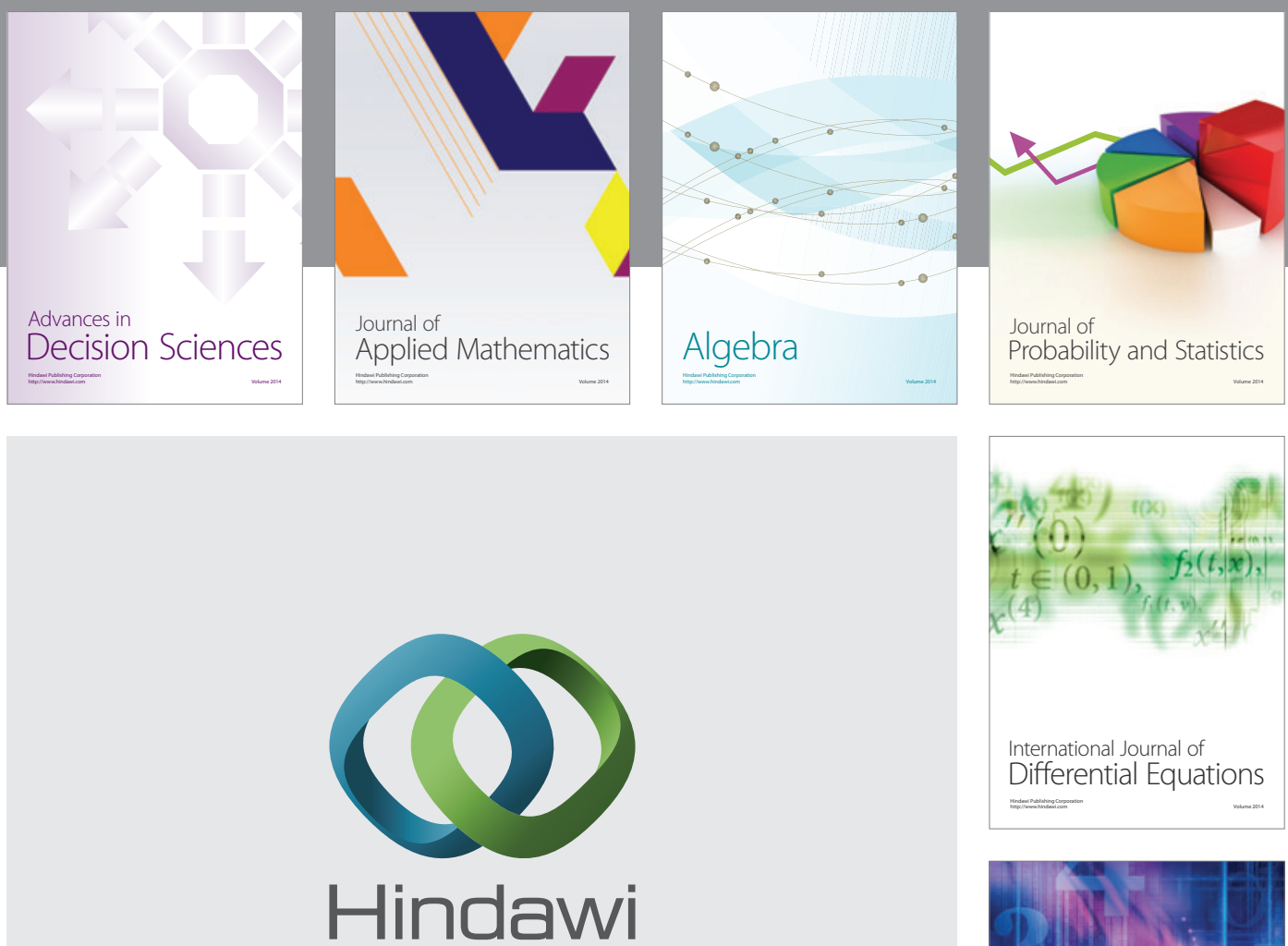

Submit your manuscripts at http://www.hindawi.com
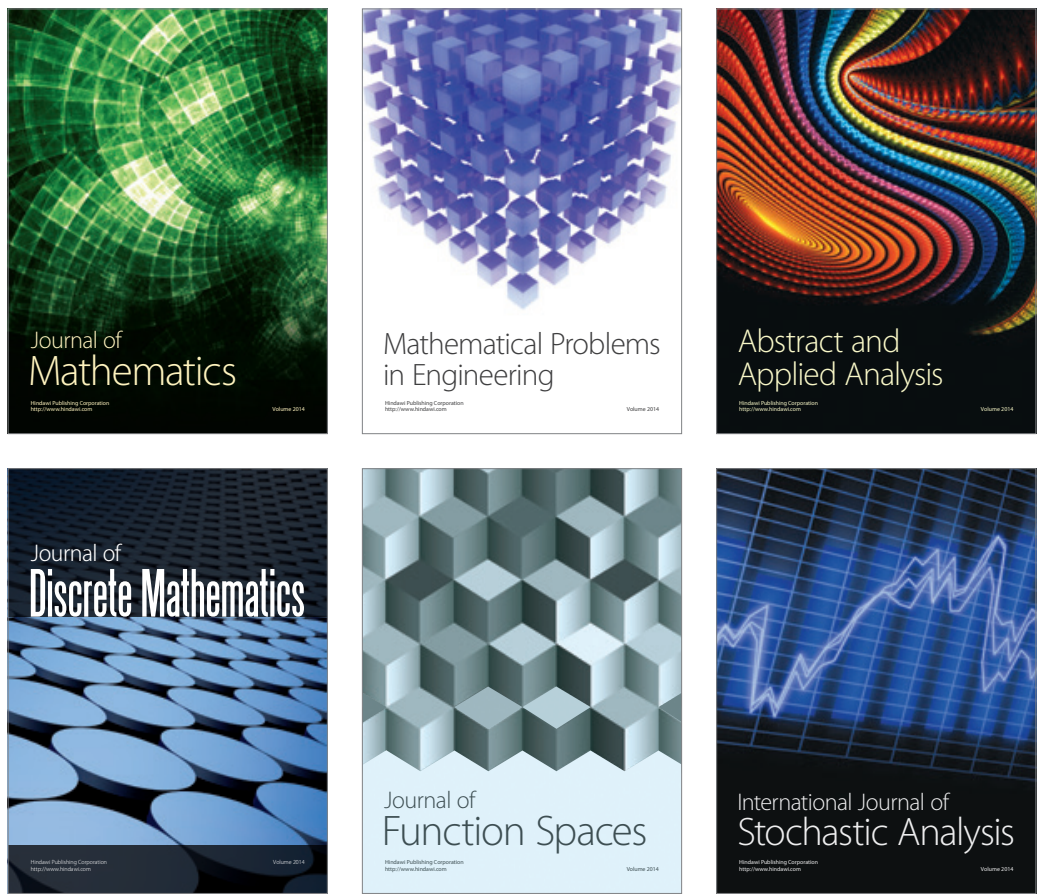

Journal of

Function Spaces

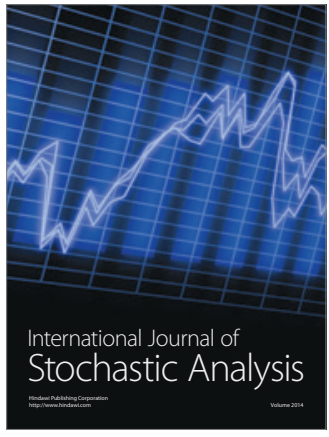

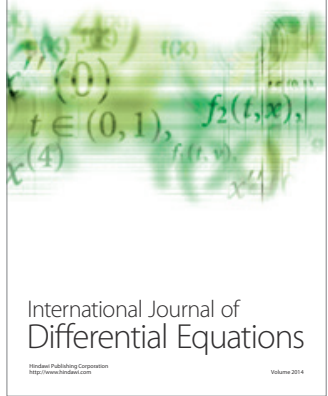
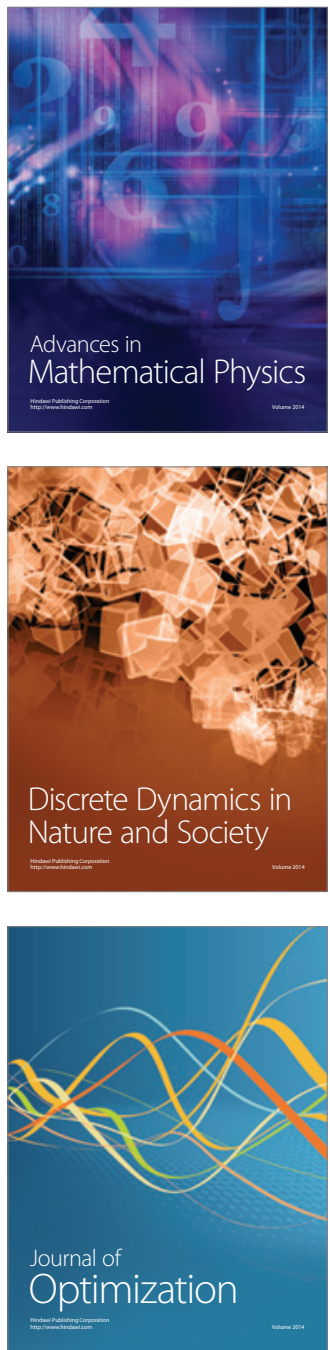